\title{
Design of low-offset low-power CMOS amplifier for biosensor application
}

\author{
Jin-Yong Zhang ${ }^{1,2}$, Lei Wang ${ }^{1}$, Bin $\mathrm{Li}^{2}$ \\ ${ }^{1}$ Institute of Biomedical and Health Engineering, Shenzhen Institutes of Advanced Technology, Chinese Academy of Sciences, \\ Shenzhen, China; ${ }^{2}$ School of Electronic and Information Engineering, South China University of Technology, Guangzhou, \\ China. \\ Email: jy.zhang@sub.siat.ac.cn; wang.lei@siat.ac.cn
}

Received 19 June 2009; revised 10 July 2009; accepted 13 July 2009.

\begin{abstract}
A compacted and low-offset low-power CMOS amplifier for biosensor application is presented in this paper. It includes a low offset Op-Amp and a high precision current reference. With a novel continuoustime DC offset rejection scheme, the IC achieves lower offset voltage and lower power consumption compared to previous designs. This configuration rejects large DC offset and drift that exist at the skin-electrode interface without the need of external components. The proposed amplifier has been implemented in SMIC 0.18- $\mu \mathrm{m}$ 1P6M CMOS technology, with an active silicon area of $100 \mu \mathrm{m}$ by $120 \mu \mathrm{m}$. The back-annotated simulation results demonstrated the circuit features the systematic offset voltage less than $80 \mu \mathrm{V}$, the offset drift about $0.27 \mu \mathrm{V} /{ }^{\circ} \mathrm{C}$ for temperature ranging from $-30^{\circ} \mathrm{C}$ to $100^{\circ} \mathrm{C}$ and the total power dissipation consumed as low as $37.8 \mu \mathrm{W}$ from a $1.8 \mathrm{~V}$ single supply. It dedicated to monitor low amplitude biomedical signals recording.
\end{abstract}

Keywords: Biomedical Integrated Circuit; CMOS Amplifier; Low-Offset and Low-Power; DC Offset Rejection; Biomedical Sensor

\section{INTRODUCTION}

Recently, there is increasing demand for portable and wearable devices to continuously monitor vital signals such as electroencephalography (EEG) and electrocardiography (ECG), blood pressure, etc. [1]. These devices usually contain various types of biosensors. CMOS amplifier is a crucial block at the front-end of these sensors, because most biomedical signals are characterized by their relative weak amplitude and low frequency, usually of few $\mathrm{mV}$ or less and the frequency below $1 \mathrm{kHz}$ [2]. Meanwhile, these signals are often accompanied by large DC offset caused by skin-electrode interface. Therefore, amplifying such weak signals requires an amplifier with low-offset and low-offset drift, which is quite challenging without using any trimmed components.

There are some techniques have been developed to deal with the design challenges. Alternatively, auto-zeroing (AZ), correlated double sampling (CDS) and chopper stabilization techniques (CHS) $[3,4,5,6]$ are utilized in sensor amplifier design to obtain DC offset rejection and high noise performance. However, these circuits have some disadvantages such as the employment of large capacitors, either off-chip or on-chip. Furthermore, these circuits add many CMOS switches that inevitably introduce switching noise, thermal noise, residual nonlinear switch errors and the CHS circuit consumes more power as circuit working in the chopping frequency. In fact these circuits were optimized for low flicker noise at the cost of higher bandwidth and worse thermal noise performance [7]. Trimming amplifier's components is another skill, but performance of this circuit is strongly related to the on-chip components matching and it increases the cost.

As a result, we turned to the CMOS amplifier design using continuous-time technique for high performance and low-cost solution. In this paper, an integrated continuous-time CMOS amplifier with low-offset voltage and low-power consumption was designed to meet the required biosensor. The proposed amplifier, designed in SMIC 0.18- $\mu \mathrm{m}$ CMOS technology, achieved less than 80 $\mu \mathrm{V}$ offset voltage and consumed only $37.8 \mu \mathrm{W}$ under a $1.8 \mathrm{~V}$ supply. It is a good candidate for biosensor application.

\section{CIRCUIT IMPLEMENTATION}

Amplifier is an important block at the front-end of the biosensor system as in [8]. Figure 1 shows the architectture of the integrated CMOS amplifier. It consists basically of three blocks, which are current reference, bias generator and low-offset amplifier core. A high precision 


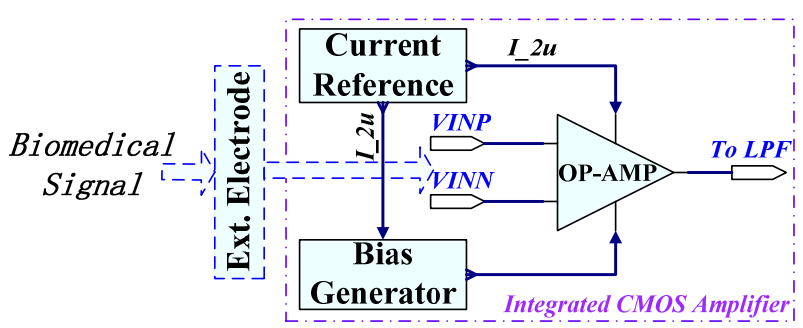

Figure 1. Architecture of the proposed integrated CMOS amplifier.

current reference was integrated in this design, it generated multiple branches of $2 \mu \mathrm{A}$ temperature and supply independent current and were used to bias the amplifier and the bias generator. The complete schematic diagram of the proposed integrated CMOS amplifier is depicted in Figure 2.

\subsection{Current Reference/Bias Generator}

Minimizing the variation of the reference current and bias voltage for amplifier is crucial as well as achieving high performance in sensor systems. A novel compensation scheme for supply and temperature dependency of MOSFET-only current reference was presented in this design [9]. The complete schematic of the reference is shown in Figure 2. It includes start up circuit, self-based current generator, supply and temperature compensation circuit.

The operation principle is that if two current outputs having the same dependency on supply voltage and temperature are subtracted with proper weighting, the compensation output would be obtained. As demonstrated in Figure 3, two self-biased current references generated $\mathrm{I}_{\text {out } 1}$ and $\mathrm{I}_{\text {out } 2}$, respectively.

$$
I_{\text {out } 1,2}=\frac{2}{\mu_{p} C_{o x}(W / L)_{p}} \cdot \frac{1}{R_{s}^{2}} \cdot\left(1-\frac{1}{\sqrt{K_{1,2}}}\right)^{2}
$$

Two current mirrors are adopted in this circuit to copy the currents $I_{\text {out } 1}$ and $I_{\text {out } 2}$ to get $I_{m 1}$ and $I_{m 2}$. The size of the transistors and the resistance $R_{S}$ are determined so the two current outputs $I_{\mathrm{m} 1}$ and $I_{\mathrm{m} 2}$ have the same supply dependency and different magnitude. Then, by subtract- ing $I_{m 2}$ from $I_{m 1}$, the supply independent output current $\mathrm{I}_{\mathrm{S}}$ could be obtained, but it is still a function of temperature. Through a simple analysis, the supply compensated output current $\mathrm{I}_{\mathrm{S}}$ was given by:

$$
I_{\text {out }, 2}=\frac{2}{\mu_{p} C_{o x}(W / L)_{p}} \cdot \frac{1}{R_{s}^{2}} \cdot\left[\left(1-\frac{1}{\sqrt{K_{1}}}\right)^{2}-\left(1-\frac{1}{\sqrt{K_{2}}}\right)^{2}\right]
$$

For the negative temperature coefficients of resistor $\mathrm{R}_{\mathrm{S}}$ and $\mu_{\mathrm{P}}$, the supply independent current $\mathrm{I}_{\mathrm{S}}$ has a proportional-to-absolute-temperature (PTAT) characteristic. The drain current of PMOSFET, $\mathrm{I}_{\mathrm{T}}$, its temperature coefficient is also positive. Then the temperature compensated output current $\mathrm{I}_{\mathrm{REF}}$ could be obtained by subtracting $\mathrm{I}_{\mathrm{T}}$ from $\mathrm{I}_{\mathrm{S}}$. In bias circuit, the master biasing current and voltage of the complete amplifier were derived from the supply and temperature independent current reference.

\subsection{Low-Offset Operational Amplifier}

Offset in operational amplifier originates in both random and systematic manner [10]. The random offset comes from imperfect fabrication of identical devices. The systematic offset can be considered as errors in the design, it occurs due to the channel length modulation of transistors and the magnitudes of the offset voltages are different according to the input and output common-mode voltages [11]. In this design, a continuous-time asymmetrical differential input structure with active DC offset rejection circuit was implemented to minimize the systematic offset of the amplifier $[12,13]$.

The principle of the active DC offset rejection technique is illustrated in Figure 4, considering the amplifier connected as a unity gain following configuration, where the input swing is nearly equal to the output swing. The common-mode level of the input and the output could be detected and amplified by the DC offset rejection circuit, and changed to the feedback signals for current sinks of the amplifier. This is a negative feedback network. By adjusting the current of the current sinks, the input and the output common-mode voltage would be maintained in same level to minimize the systematic offset.

As depicted in Figure 2, the circuit of low-offset

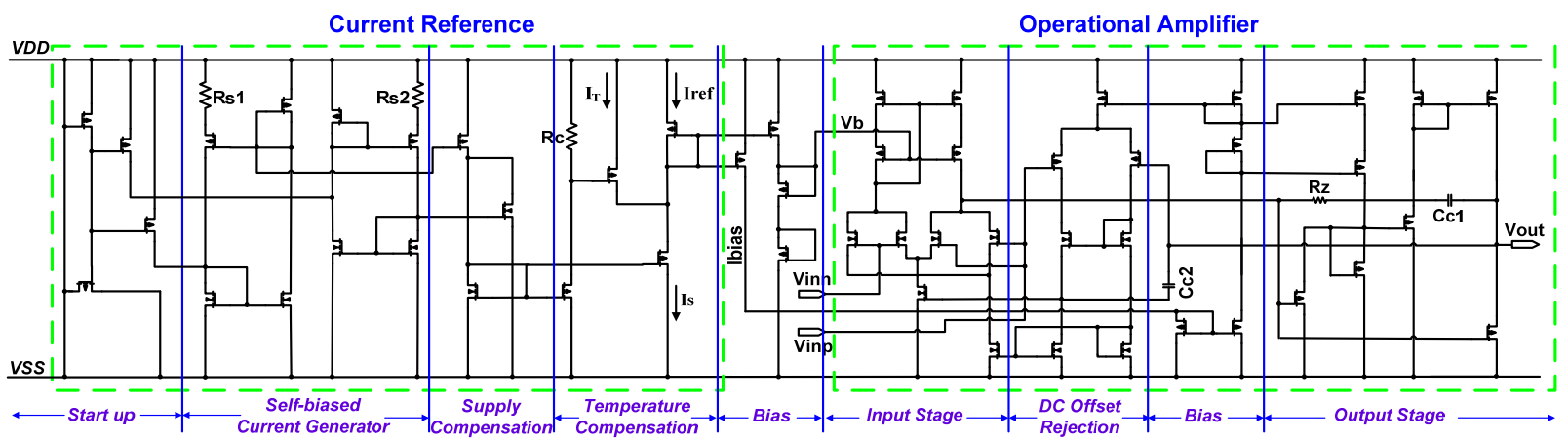

Figure 2. Complete schematic diagram of the integrated CMOS amplifier. 


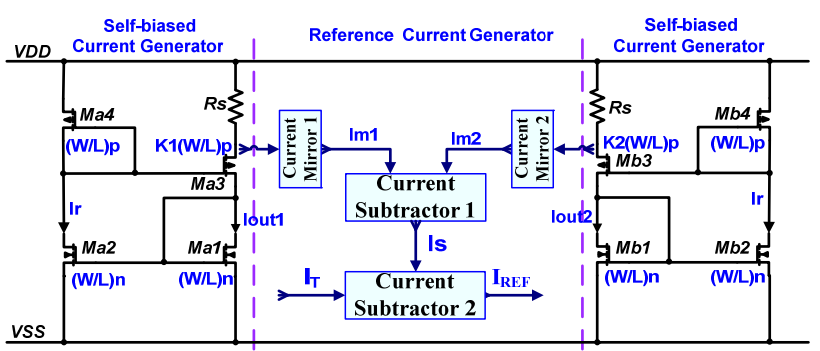

Figure 3. The basic principle of the current reference.

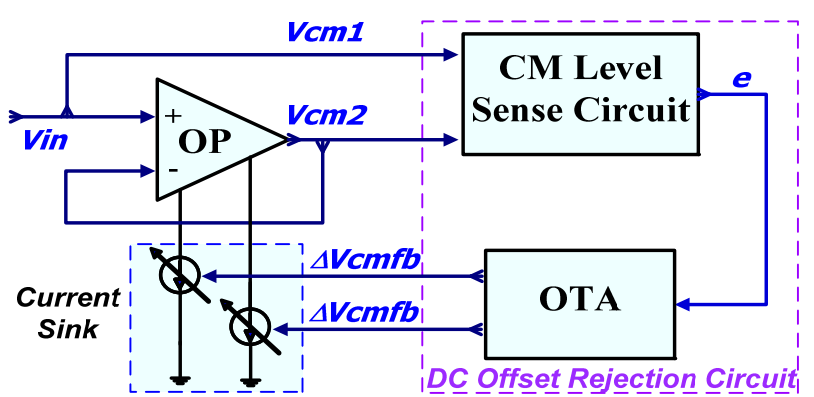

Figure 4. The principle of the DC offset rejection technique.

amplifier is divided into three parts: input stage, DC offset rejection circuit, and output stage. In input stage, the input MOS transistor pairs were designed as asymmetrical differential structure. Besides, the input transistors and active load transistors with appropriate dimensions were used in order to obtain good matching characteristic. In DC offset rejection circuit, a single stage OTA structure was adopted to amplify the difference between input and output common-mode level. Therefore, via cascading the NMOS pairs to obtain the large gain of the OTA. In output stage, the class-AB structure was designed to improve the power efficiency, openloop gain and driving capability. RC Miller compensation and capacitor compensation techniques were used in this circuit [14].

Finally, a careful layout was planned to reduce process-related random offset: a) the symmetrical layout style was addressed through the entire layout, b) common-centroid cross-coupling layout strategy together with poly guard rings were adopted for critical devices and c) input pairs, active mirror loads and current sources that need to be matched were selectively grouped and arranged with dummies to minimize the effect of spacing-dependent parameter mismatch [15].

\section{SIMULATION RESULTS AND DISCUSSIONS}

This design has been implemented using the SMIC $0.18-\mu \mathrm{m}$ CMOS 1P6M technology. Figure 5 shows the complete layout of the integrated CMOS amplifier, with total silicon area of $100 \mu \mathrm{m}$ by $120 \mu \mathrm{m}$. This chip has sent to be fabricated. We will test it with real-world physiological signals in near future.

\subsection{Current Reference}

The temperature drifts and supply regulation of the reference current are shown in Figures 6 (a) and (b), respectively. The reference offered a current of $2 \mu \mathrm{A}$ when adjusted to have a zero temperature coefficient at room temperature. It could be observed that an overall temperature coefficient of $0.625 \mathrm{nA} /{ }^{\circ} \mathrm{C}$ is obtained between $0^{\circ} \mathrm{C}$ and $80^{\circ} \mathrm{C}$, which corresponds to about $2.2 \%$ variation. The response of it is better for temperature from

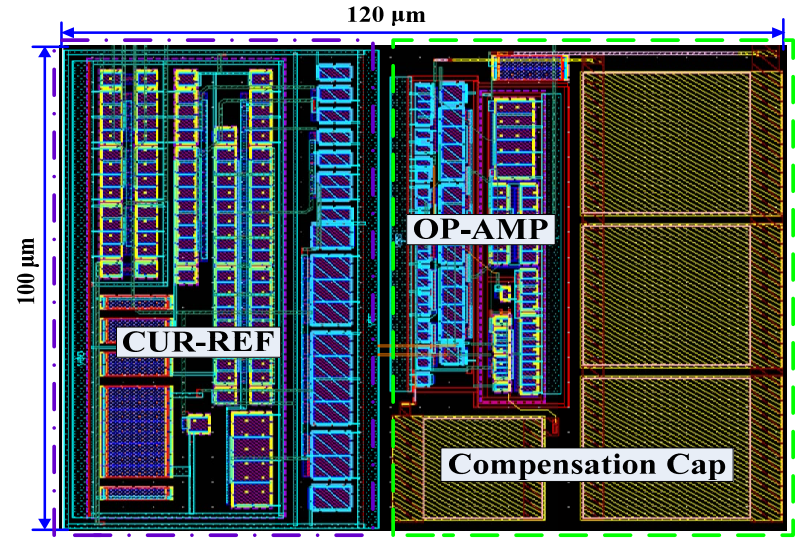

Figure 5. Layout view of the integrated CMOS amplifier.

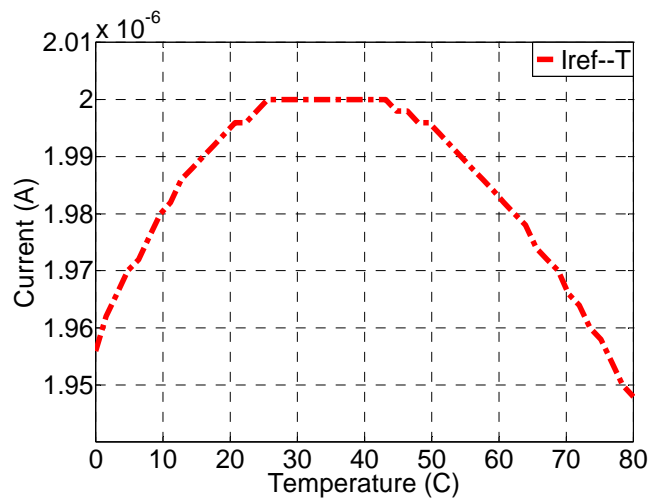

(a)

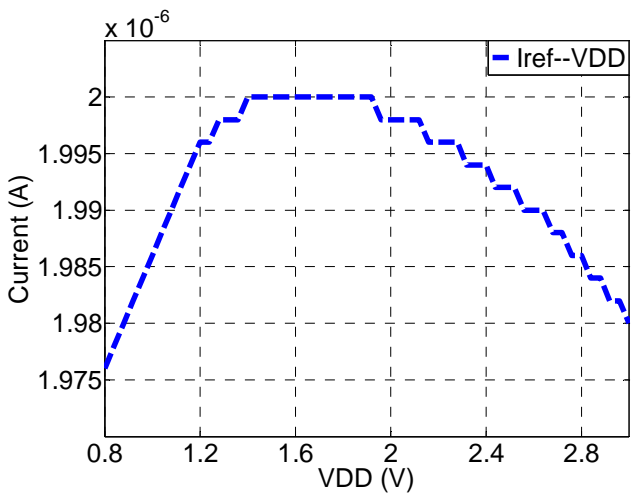

(b)

Figure 6. Layout view of the integrated CMOS amplifier Simulated reference current dependence on temperature (a) and supply voltage (b). 
$20^{\circ} \mathrm{C}$ to $50^{\circ} \mathrm{C}$ with a temperature coefficient of $0.13 \mathrm{n}$ $\mathrm{A} /{ }^{\circ} \mathrm{C}$. The line regulation of reference current is about $0.55 \% / \mathrm{V}$ when the supply voltage ranges from $0.8 \mathrm{~V}$ to $3 \mathrm{~V}$.

\subsection{Low-Offset Operational Amplifier}

Figure 7 shows the $\mathrm{AC}$ responses of the integrated CMOS amplifier while driving a $3 \mathrm{pF}$ capacitive load. It offered $60 \mathrm{~dB}$ open-loop gain, $63.5^{\circ}$ phase margin, and 2.82 MHz unity gain bandwidth. DC sweep analysis of the amplifier connected in an inverting unity-gain configuration is shown in Figure 8. The simulation results showed good following characteristic between Vin and Vout, and the offset voltage less than $80 \mu \mathrm{V}$ by averaging. Figure 9 depicts the offset drifts of the amplifier over a wide temperature range from $-30^{\circ} \mathrm{C}$ to $100^{\circ} \mathrm{C}$. The mean offset drift is $0.24 \mu \mathrm{V} /{ }^{\circ} \mathrm{C}$, it illustrated the integrated CMOS amplifier was able to sustain low offset voltage over a wide temperature range.

The performance achieved in this design was compared with other state-of-the-art designs for biomedical application. As listed in Table 1, it could be seen that our design offered comparable performances. The proposed

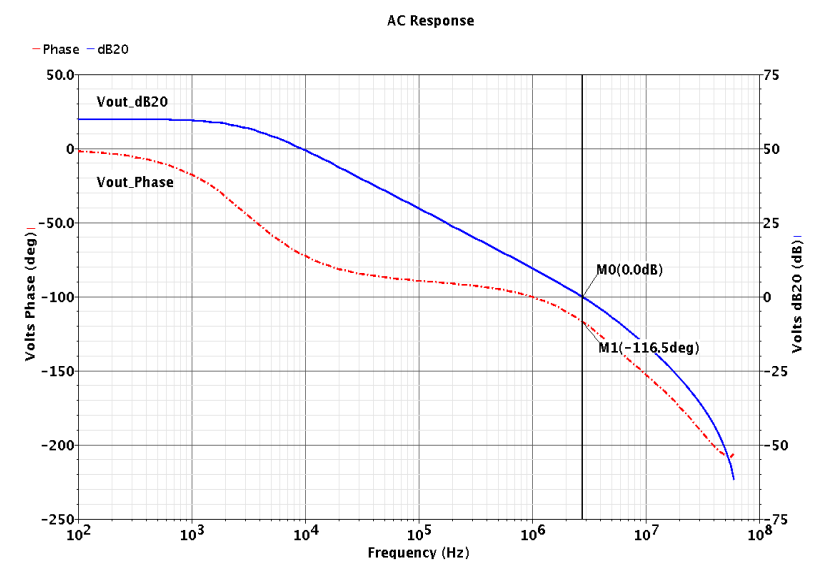

Figure 7. AC simulation results of the integrated CMOS amplifier.

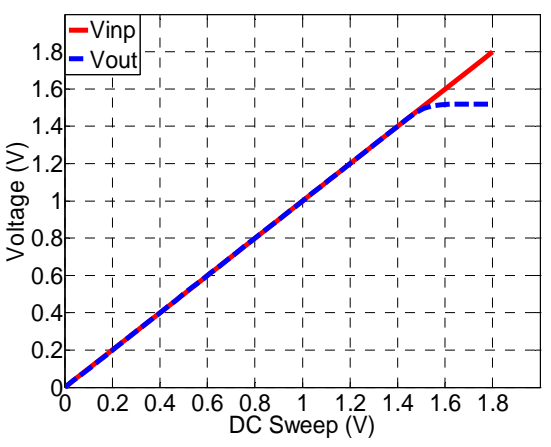

(a)

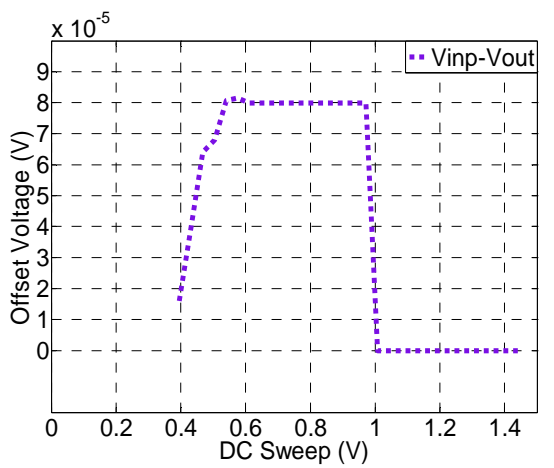

(b)

Figure 8. The simulation results of the following characteristic (a)and offset tunning range (b).

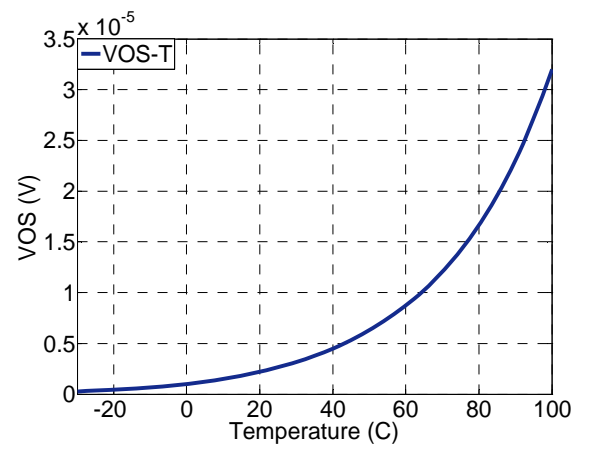

Figure 9. The offset variation with temperature.

Table 1. Performance comparison with other publications. (*instrumentation amplifier)

\begin{tabular}{cccccc}
\hline Parameter & Ref [5] 2002 & Ref [16] 2004 & Ref [17] $]^{*} \mathbf{2 0 0 5}$ & Ref [18] 2007 & This work \\
\hline CMOS Technology & $0.7-\mu \mathrm{m}$ & $1.5-\mu \mathrm{m}$ & $0.35-\mu \mathrm{m}$ & $0.18-\mu \mathrm{m}$ & $0.18-\mu \mathrm{m}$ \\
Supply & $5 \mathrm{~V}$ & $3 \mathrm{~V}$ & $3 \sim 4 \mathrm{~V}$ & $1 \mathrm{~V}$ & $1.8 \mathrm{~V}$ (Typical) \\
Gain & $60.6 \mathrm{~dB}$ & $39.3 \mathrm{~dB}$ & $56 \mathrm{~dB}$ & $14 \mathrm{~dB}$ & $60 \mathrm{~dB}$ \\
Phase Margin & - & - & - & - & $63.5^{\circ}$ \\
Unity Gain Bandwidth & $5.5 \mathrm{kHz}$ & $2.7 \mathrm{KHz}$ & $130 \mathrm{KHz}$ & $2.7 \mathrm{KHz}$ & $2.82 \mathrm{MHz}$ \\
CMRR & $137 \mathrm{~dB}$ & - & $100 \mathrm{~dB}(@ 60 \mathrm{~Hz})$ & - & $>85 \mathrm{~dB}(@ \mathrm{DC}-100 \mathrm{kHz})$ \\
PSRR & - & $50 \mathrm{~dB}$ & - & $50 \mathrm{~dB}$ & $>100 \mathrm{~dB}(@ \mathrm{DC}-10 \mathrm{kHz})$ \\
Positive Slew Rate & - & $0.64 \mathrm{~V} / \mu \mathrm{s}$ & $50 \mathrm{mV} / \mu \mathrm{s}$ & $10 \mathrm{mV} / \mu \mathrm{s}$ & $3.45 \mathrm{~V} / \mu \mathrm{s}$ \\
Negative Slew Rate & - & $0.64 \mathrm{~V} / \mu \mathrm{s}$ & $50 \mathrm{mV} / \mu \mathrm{s}$ & $10 \mathrm{mV} / \mu \mathrm{s}$ & $1.67 \mathrm{~V} / \mu \mathrm{s}$ \\
Input Offset Voltage & $88.7 \mu \mathrm{V}$ & $\sim 811 \mu \mathrm{V}$ & $0.3 \mathrm{mV}$ & $1.7 \mathrm{mV}$ & $80 \mu \mathrm{VV}(\mathrm{max})$ \\
Offset Drift & - & - & - & - & $0.27 \mu \mathrm{VV} /{ }^{\circ} \mathrm{C}$ \\
Power Dissipation & $11 \mathrm{~mW}$ & $114.8 \mu \mathrm{W}$ & $72.6 \mu \mathrm{W}$ & $3.15 \mu \mathrm{W}$ & $37.8 \mu \mathrm{W}$ \\
Core Area & $5 \mathrm{~mm}^{2}$ & $0.107 \mathrm{~mm}^{2}$ & $0.2 \mathrm{~mm}$ & $0.056 \mathrm{~mm}{ }^{2}$ & $0.012 \mathrm{~mm}{ }^{2}$ \\
\hline
\end{tabular}


CMOS amplifier performed technical merits of lowoffset voltage, reasonable low-power and with relative small die size, confirming the effectiveness and robustness of the proposed circuit architecture when using both circuit design technique and careful layout technique.

\section{CONCLUSIONS}

A low-offset low-power and compacted CMOS amplifier with continues-time active DC offset rejection design technique for biosensor applications is presented on-chip in this paper, without the need of trimming. To improve circuitry robustness over power supply and temperature, a high precision current reference was integrated in this design. The whole circuit occupies an area of $100 \mu \mathrm{m}$ by $120 \mu \mathrm{m}$. The back-annotated simulation results suggested that this integrated CMOS amplifier can offer significantly enhanced metrics, in terms of the low-offset less than $80 \mu \mathrm{V}$, the offset drift about $0.27 \mu \mathrm{V} /{ }^{\circ} \mathrm{C}$ for temperature ranging from $-30^{\circ} \mathrm{C}$ to $100^{\circ} \mathrm{C}$, and the total power dissipation approximately $37.8 \mu \mathrm{W}$ at a single 1.8 $\mathrm{V}$ power supply. This integrated CMOS amplifier is particularly useful for a wide range of biosensor applications where a front-end preamplifier is required.

\section{REFERENCES}

[1] Yazicioglu, R. F., Merken, P., and Van Hoof, C., (2005) Integrated low-power 24-channel EEG front-end, Electronics Letters, 41(8), 457-458.

[2] Webster, J. G., (1998) Medical instrumentation: Application and design, 3rd Edition, Wiley, NewYork.

[3] Chan, C. H., Wills, J., LaCoss, J., Granacki, J. J., and Choma, J., (2007) A novel variable-gain micro-power band-pass auto-zeroing CMOS Amplifier, IEEE International Symposium Circuits and Systems, 337-340,.

[4] Permanod, B., Silawan, N., and Toumazou, C., (2007) Drift reduction in Ion-sensitive FETs using correlated double sampling, IET Electronics Letter, 43(16), 857859.

[5] Panitantum, N. and Wongkomet, N., (2002) A low-offset high-CMRR switched-capacitor instrumentation amplifier for data acquisition applications, Circuits and Systems, 1, 339-342.

[6] Hanasusanto, G. A. and Zheng, Y. J., (2007) A chopper stabilized pre-amplifier for biomedical signal acquisition, International Symposium on Integrated Circuits, 200-203.
[7] Li, L., (2007) High gain low power operational amplifier design and compensation techniques, A Dissertation of department of Electrical and Computer Engineering, Brigham Young University.

[8] Teo, T. H., Lim, G. K., David, D. S., Tan, K. H., Gopalakrishnan, P. K., kHz-s.l, and Singh, R., (2007) Ultra low-power sensor node for wireless health monitoring system, IEEE International Symposium on Circuits and Systems, 2363-2366.

[9] Yoo, C. A. and Park, J., (2007) CMOS current reference with supply and temperature compensation, Electronics Letters, 43(25), 1422-1424.

[10] Chan, P. K., Siek, L., Tay, H. C., and Su, J. H., (2000) A low-offset class-AB CMOS operational amplifier, in Proceedings, The 2000 IEEE International Symposium on Circuits and Systems, 3, 455-458.

[11] Holzmann, P. J., Wiegerink, R. J., Gierkink, S. L. J., Wassenaar, R. F., and Stroet, P., (1996) A low-offset low-voltage CMOS op amp with rail-to-rail input and output ranges, 1996 IEEE International Symposium on Circuits and Systems, 1, 179-182.

[12] Lim, K. T., Kim, S. J., and Kwon, O. K., (2003) The OP-amplifier with offset cancellation circuit, Electron Devices and Solid-State Circuits, 2003 IEEE Conference, 445-447.

[13] Gosselin, B., Ayoub, A. E., and Sawan, M., (2006) A low-power bioamplifier with a new active DC rejection scheme, in Proceedings, IEEE International Symposium on Circuits and System, 2185-2188.

[14] Hogervorst, R., Tero, J. P., Eschauzier, R. G. H., and Huijsing, J. H., (1994) A compact power-efficient $3 \mathrm{~V}$ CMOS rail-to-rail input/output operational amplifier for VLSI cell libraries, Solid-State Circuits, IEEE Journal, 29(12), 1505-1513.

[15] Christopher Saint and Judy Saint, IC Mask Design: Essential Layout Techniques, McGraw-Hill Professional, 2002.

[16] Mohseni, P. and Najafi, K., (2004) A fully integrated neural recording amplifier with DC input stabilization, Biomedical Engineering, IEEE Transactions, 51(5), 832837.

[17] Maryam, S. B., Rakesh, K. L., and Dinesh, K., (2005) A low-power and compact analog CMOS processing chip for portable ECG recorders, Asian Solid-State Circuits Conference, 473-476.

[18] Putra, A., Teo, T. H., and Rajinder, S., (2007) Ultra low-power low-voltage integrated preamplifier using class-AB op-amp for biomedical sensor application, International Symposium on Integrated Circuits, 216-219. 